University of Wollongong

Research Online

Australian Institute for Innovative Materials -

Papers

Australian Institute for Innovative Materials

$1-1-2014$

Fabrication of graphene foam supported carbon nanotube/polyaniline hybrids for high-performance supercapacitor applications

Hongxia Yang

Zhengzhou University

Nan Wang

Zhengzhou University

Qun Xu

Zhengzhou University

Zhimin Chen

Zhengzhou University

Yumei Ren

Zhengzhou University

See next page for additional authors

Follow this and additional works at: https://ro.uow.edu.au/aiimpapers

Part of the Engineering Commons, and the Physical Sciences and Mathematics Commons

Research Online is the open access institutional repository for the University of Wollongong. For further information contact the UOW Library: research-pubs@uow.edu.au 


\title{
Fabrication of graphene foam supported carbon nanotube/polyaniline hybrids for high-performance supercapacitor applications
}

\author{
Abstract \\ A large-scale, high-powered energy storage system is crucial for addressing the energy problem. The \\ development of high-performance materials is a key issue in realizing the grid-scale applications of \\ energy-storage devices. In this work, we describe a simple and scalable method for fabricating hybrids \\ (graphenepyrrole/ carbon nanotube-polyaniline (GPCP)) using graphene foam as the supporting template. \\ Graphene-pyrrole (G-Py) aerogels are prepared via a green hydrothermal route from two-dimensional \\ materials such as graphene sheets, while a carbon nanotube/polyaniline (CNT/PANI) composite \\ dispersion is obtained via the in situ polymerization method. The functional nanohybrid materials of \\ GPCP can be assembled by simply dipping the prepared G-py aerogels into the CNT/PANI dispersion. The \\ morphology of the obtained GPCP is investigated by scanning electron microscopy (SEM) and \\ transmission electron microscopy (TEM), which revealed that the CNT/PANI was uniformly deposited \\ onto the surfaces of the graphene. The as-synthesized GPCP maintains its original three-dimensional \\ hierarchical porous architecture, which favors the diffusion of the electrolyte ions into the inner region of \\ the active materials. Such hybrid materials exhibit significant specific capacitance of up to $350 \mathrm{~F} \mathrm{~g}^{-1}$, \\ making them promising in large-scale energy-storage device applications.

\section{Keywords} \\ polyaniline, fabrication, hybrids, graphene, high, performance, supercapacitor, applications, foam, \\ supported, carbon, nanotube

\section{Disciplines} \\ Engineering | Physical Sciences and Mathematics

\section{Publication Details} \\ Yang, H., Wang, N., Xu, Q., Chen, Z., Ren, Y., Razal, J. M. \& Chen, J. (2014). Fabrication of graphene foam \\ supported carbon nanotube/polyaniline hybrids for high-performance supercapacitor applications. 2D \\ Materials, 1 (3), 1-14.

\section{Authors} \\ Hongxia Yang, Nan Wang, Qun Xu, Zhimin Chen, Yumei Ren, Joselito M. Razal, and Jun Chen
}




\section{Fabrication of Graphene Foam supported Carbon}

\section{Nanotube/Polyaniline hybrids for High-Performance}

\section{Supercapacitor Applications}

Hongxia Yang ${ }^{1}$, Nan Wang ${ }^{1}$, Qun Xu ${ }^{1 *}$, Zhimin Chen ${ }^{1}$, Yumei Ren ${ }^{1}$, Joselito M. Razal, ${ }^{2}$ Jun $\mathrm{Chen}^{3 *}$

${ }^{1}$ College of Materials Science and Engineering, Zhengzhou University, Zhengzhou 450052, P. R. China

2 Institute for Frontier Materials, Deakin University, Geelong VIC 3216, Australia.

${ }^{3}$ Intelligent Polymer Research Institute, ARC Centre of Excellence for Electromaterials Science, Australian Institute of Innovative Materials, University of Wollongong, New South Wales 2522, Australia

Abstract

Large scale energy storage system with high power is crucial for addressing the energy problem. The development of high-performance materials is a key issue in realizing grid-scale applications of energy storage devices. In this work, we describe a simple and scalable method to fabricate hybrids (graphene-pyrrole/carbon nanotube-polyaniline (GPCP) using graphene foam as the supporting template. Graphene-pyrrole (G-Py) aerogels are prepared by a green hydrothermal route from 2D materials - graphene sheets, while CNT/PANI composite dispersion is obtained via in-situ polymerization method. The functional nanohybrid materials of GPCP can 
be assembled together by simply dipping the prepared G-py aerogels into the CNT/PANI dispersion. The morphology of the obtained GPCP are investigated by scanning electron microscopy (SEM) and transmission electron microscope (TEM), which revealed that CNT/PANI were uniformly deposited onto the surfaces of graphene. The as-synthesized GPCP maintains the original three-dimensional (3D) hierarchical porous architecture, which favor the diffusion of the electrolyte ions into the inner region of active materials. Such hybrid materials exhibit significant specific capacitance of up to $350 \mathrm{~F} \mathrm{~g}^{-1}$, making them promising in large-scale energy storage device applications.

Keywords: Graphene; carbon nanotube; polyaniline; supercapacitor; 


\section{Introduction}

Constructing three-dimensional (3D) graphene-based hybrid nanostructures with anticipated function has become one of the most active research fields in the past several years. The integration of individual 2D graphene sheets into a 3D macroscopic assembly and then into a functional system is an attractive strategy for realizing large scale applications. Lightweight graphene aerogel composed of interconnected 3D networks has attracted intensive attention because of their unique physical properties, such as low density, high electrical conductivity, rich

porosity, and specific surface area [1,47-49]. These properties have caused rapid advance of various applications, extending from supercapacitors [2,3], stretchable electronics [4,5], catalysis [6,7], hydrogen storage [8], to environmental remediation [9]. Despite the aforementioned success, graphene in their pristine form are insufficient to satisfy diverse specific demands for practical applications, especially for large scale grid applications.

Carbon materials [10-13], transition metal oxides [14] and conducting polymers [15-18] are the three main types of greatly investigated electrode materials. Recently, polyaniline (PANI) has drawn much attention in electrochemical studies owing to its excellent properties such as low cost, ability to easily form various nanostructures, good biocompatibility and environmental stability $[19,20]$. However, PANI suffers from the mechanical degradation problem caused by swelling and shrinkage during the doping and dedoping process [21,22], leading to limited cycling life of PANI-based supercapacitors. In order to overcome these limitations, much effort has been devoted to preparing hybrid electrodes by combining PANI with other nanomaterials. To date, various attempts have been made to integrate PANI and carbon nanotubes $[23-25,50]$ to obtain nanocomposites with excellent electrochemical properties and improved stability [26-28]. 
In this work, highly porous graphene scaffolds containing pyrrole (Py) with 3D intercrossed structures were synthesized via a green hydrothermal route (the resulting material is referred to as G-Py aerogel). CNT/PANI composite solution was prepared using a simple in-situ polymerization method. The GPCP hybrid materials are then obtained via dipping the G-Py foam into the CNT/PANI dispersion. The incorporation of CNT/PANI into the porous structures of graphene-based foam could prevent aggregation/restacking of graphene sheets to miantian the foam architectures during the following treatment and testing processes. Also, the well deposition of PANI nanorods on CNT effectively increases the electrochemical utilization of PANI, and reduces the ion diffusion path during charge-discharge process. Moreover, CNTs serve as conducting wires to provide electronic transport paths for the conductive networks inside the bulk electrode matrix and improve the stability of GPCP hybrid materials upon electrochemical cycling. The as-prepared GPCP hybrid porous electrodes have the significant potential to be used as promising 3D electrode materials for lightweight and flexible energy storage devices with stable high performance.

\section{Experimental}

2.1 Chemicals. All analytical grade reagents were used without further purification. Aniline (ANI, Tianjin Damao Chemical Reagent Factory), Pyrrole (Py, Aladdin), ammonium persulfate (APS, Sigma Aldrich) and carbon nanotubes (CNT, Beijingdekedaojing Co., Ltd, China).

2.2 Preparation of Graphene-Py aerogel. GO was prepared by oxidation of natural graphite powder according to the modified Hummers' method [29,30]. We first prepared 3D graphene by hydrothermal treatment of $6 \mathrm{ml} 2 \mathrm{mg} \mathrm{mL}^{-1}$ homogeneous graphene oxide (GO) aqueous dispersion with 5 vol\% pyrrole (Py) in a Teflon-lined autoclave at $180{ }^{\circ} \mathrm{C}$ for $12 \mathrm{~h}$. Then the 
autoclave was naturally cooled to room temperature and the product was freeze-dried for $12 \mathrm{~h}$ to obtain the G-Py aerogel [31].

2.3 Preparation of CNT/PANI solution. The CNT/PANI solution was synthesized by chemical oxidative polymerization in an ice-water bath. In-situ chemical polymerization was conducted according to the following procedure. MWNT $(6 \mathrm{mg})$ were dispersed in $15 \mathrm{ml} 1 \mathrm{M}$ $\mathrm{HClO}_{4}$ for $2 \mathrm{~h}$ under sonication. Then, different amounts of ANI (from 20 to $50 \mathrm{mM}$ ) were dispersed into the mixture under the protection of $\mathrm{N}_{2}$. Ethanol $(500 \mu \mathrm{L})$ was added into the mixture to prevent the mixture from freezing. $5 \mathrm{ml}$ of $1 \mathrm{M} \mathrm{HClO}_{4}$ solution containing APS (the molar ratio of ANI to APS was $3: 1$ ) was added dropwise into the reaction mixture and mixed for $7 \mathrm{~h}$. The dark green product was collected by centrifugation and washed with large amount of water/ethanol solution (1:1 volume ratio). The products were dried in vacuum for $12 \mathrm{~h}$.

2.4 Preparation of Graphene-Py/CNT/PANI hybrid. The as-prepared Graphene-Py aerogel (20 mg) was dipped into as-prepared CNT/PANI ethanol solution $(5 \mathrm{ml})$ with various PANI concentration for $2 \mathrm{~h}$ and dried in vacuum for $12 \mathrm{~h}$. The final Graphene-Py/CNT/PANI samples were referred to as GPCP-X where $\mathrm{X}=20,30,40,50$ representing the aniline monomer concentration during the preparation of CNT/PANI composites.

2.5 Characterization. The morphologies of the obtained structures were characterized by field-emission scanning electron microscope (FE-SEM, JSM 7500F) and transmission electron microscopy (TEM) (JEM-2100). X-ray diffraction (XRD) patterns of samples were measured on a Y-2000 X-ray Diffractometer with copper K $\alpha$ radiation $(\lambda=1.5406 \AA)$ operating at $40 \mathrm{kV}$ and 40 mA. The Raman measurements were carried out on a Renishaw Microscope System RM2000 with a $50 \mathrm{mWAr}+$ laser at $514.5 \mathrm{~nm}$. Fourier transform infrared spectra (FTIR) were recorded on a TENSOR 27 FTIR spectrometer (Bruker) in the absorption mode with resolution of $2 \mathrm{~cm}^{-1}$. X- 
ray photoelectron spectroscopy (XPS) measurements were made on a ESCALABMK spectrometer using electrostatic lens mode with a pass energy of $100 \mathrm{eV}$.

2.6 Preparation of working electrode and electrochemical measurements. The working electrodes for supercapacitors were prepared by thoroughly mixing the electroactive nanocomposites, carbon black and poly(tetrafluoroethylene) in a mass ratio of 80:10:10, respectively. The obtained slurry was coated and pressed onto a foam nickel to prepare a working electrode, and then dried at $80{ }^{\circ} \mathrm{C}$ for $24 \mathrm{~h}$ in a vacuum oven. The electrochemical performance of the as-prepared hybrid composites as working electrodes was carried out on a CHI660D electrochemical station in a three-electrode configuration with platinum foil and $\mathrm{Ag} / \mathrm{AgCl}$ as counter and reference electrode, respectively. The cyclic voltammetry and galvanostatic charging/discharging techniques were employed to investigate the electrochemical performance of the composites. The applied potential window ranged from $-1 \mathrm{~V}$ to $0 \mathrm{~V}$ in $1 \mathrm{M}$ $\mathrm{KOH}$ electrolyte.

\section{Results and Discussion}

A schematic illustration of the fabrication process for the nanocomposites is depicted in Figure 1. The GPCP-X nanohybrids were prepared by simply dipping the G-Py aerogel (prepared via a one-step hydrothermal method) into the CNT/PANI nanocomposites solution (prepared via in-situ polymerization).

\subsection{Morphology and structure characterization of GPCP-X.}

Small graphene sheets are usually constructed or assembled into macroscopic materials for practical applications. The critical issue typically encountered in this process is the irreversible aggregation or restacking of graphitic structures. In this work, pyrrole was introduced to prevent aggregation of the graphene sheets. Shown in Figure 2a are the G-Py hydrogel and aerogel. The 
density of the G-Py aerogel is $12 \mathrm{mg} \mathrm{cm}^{-3}$. The corresponding SEM investigation revealed that G-Py possesses 3D interconnected framework with randomly opened macropores. Considering the conjugated structure of pyrrole with its electron-rich $\mathrm{N}$ atom, it can attach to the surfaces and galleries of GO sheets through hydrogen-bonding and/or $\pi-\pi$ interactions. During the hydrothermal process, pyrrole works as a swelling agent to effectively prevent GO from selfstacking, thereby obtaining a graphene aerogel with larger volume than pure graphene hydrogel. Figures S1b and c illustrates that G-Py had a loose structure with much thinner connection walls compared to pure graphene hydrogel [31]. The pore sizes of the G-Py network range from submicrometer to several micrometers [32]. The interconnected macropores within the GO framework are favorable for the transfer of buffering ions for they minimize the diffusion distance from the external electrolyte to the interior surfaces [33]. The morphology of the asprepared materials was further observed by TEM. The curled and wrinkled morphology of graphene sheets indicate the porous structure of the obtained G-Py [34], which is in agreement with SEM images. The FTIR spectra of the G-Py aerogel, pyrrole, and polypyrrole shown in Figure $2 \mathrm{~d}$ show the appearance of representative bands at $617,685,1407,1485$ and $1517 \mathrm{~cm}^{-1}$, which indicate the presence of Py.

The SEM images of CNT/PANI nanocomposites with different concentration of ANI (30, 40 and $50 \mathrm{mM}$ ) are shown in Figure 3 and Figure S2a, c. It can be observed that CNT/PANI nanocomposites with uniform morphology have been successfully prepared. The magnified SEM image clearly shows that the PANI nanorods align vertically on the surface of the CNT, which is due to the $\pi-\pi$ stacking interaction between the graphitic structures of CNT and the aromatic rings of PANI [35]. As seen in the Figure 3b, $\mathrm{c}$ and d, the average diameter of the CNT/PANI composites increases with increasing ANI concentration. It is apparent that the length of the 
vertical PANI nanorods grown on CNT can be controlled by changing the initial concentration of ANI, which will allow the controllable electrochemical performance of the supercapacitor [36].

The typical SEM and TEM images of the obtained GPCP nanohybrids are shown in Figure 4 and Figure S2b, d. As shown in the Figure $4 b$, c and d, it can be clearly observed that CNT/PANI was uniformly grown on the surfaces of graphene. They act as bridges between different graphene sheets to form a conductive network [37], as well as preventing the stacking of graphene sheets. In addition, the multi-composition can have synergistic effects to take advantage of both Faradaic and non-Faradaic processes for capacity-charge storage with excellent electrochemical properties [38].

Figure 5a shows the X-ray diffraction spectra of GPCP-50 nanohybrids. The X-ray data of the GPCP-50 nanohybrids show weaker crystalline peaks to that of neat PANI that has a semicrystalline structure. The main peaks in the XRD pattern of pure PANI are located at $9.0^{\circ}, 21.5^{\circ}$ and $25.3^{\circ}$, which are the characteristic peaks of PANI [39]. Compared with PANI, the XRD pattern of the GPCP-50 nanohybrids exhibits one peak $\left(21.5^{\circ}\right)$ similar to that of PANI, indicating that in the hybrids the PANI exhibits poor crystallinity because of the existence of G-Py aerogel [40].

To further study the interaction between these carbon materials and PANI, Raman spectroscopy analysis is employed. The Raman spectra of the pristine CNT, GO, G-Py, neat PANI and GPCP-X hybrids are presented in Figure 5b. The obvious peaks at about 1350 and $1600 \mathrm{~cm}^{-1}$ can be attributed to the disordered structure (D band, $\mathrm{sp}^{3}$ carbon atoms of defects and disorders) and graphitic structure ( $\mathrm{G}$ band, $\mathrm{sp}^{2}$ carbon atoms in graphitic sheets) of GO, respectively [41]. For the Raman spectrum of GPCP-50 sample, the band at about $419 \mathrm{~cm}^{-1}$ is ascribed to out-of-plane amine ring deformations and the band at $574 \mathrm{~cm}^{-1}$ is a confirmation of 
the existence of ring deformation and phenazine- or phenoxazine-like segments. The bands around 779 and $818 \mathrm{~cm}^{-1}$ is in agreement with amine and imine deformation (C-N-C bending) [42]. However, the GPCP-50 nanohybrids show sharp peaks at about $1180 \mathrm{~cm}^{-1}$ representing the $\mathrm{C}-\mathrm{H}$ bending in the benzene- or quinine-type rings. The peak at $1350 \mathrm{~cm}^{-1}$ is a clear symbol of C$\mathrm{N}^{+}$stretching, and the peak at $1485 \mathrm{~cm}^{-1}$ stands for the $\mathrm{C}=\mathrm{N}$ stretching vibration in the emeraldine base of imines due to the n-doping effect of PANI. The Raman results further confirm that the GPCP-50 nanohybrids with strong $\pi-\pi$ interactions was successfully synthesized $[43,44]$.

A surface elements analysis was carried out on the GO film and G-Py aerogel using X-ray photoelectron spectroscopy (XPS). The obtained XPS full spectrum (Figure 5c) shows signals from C, N and O elements, indicating the presence of Py on the G-Py aerogel. The peaks with lower binding energy located at 397.8 and $399.9 \mathrm{eV}$ correspond to pyridine-like and pyrrole-like nitrogen, respectively [45], as illustrated in Figure 5d. During the hydrothermal reaction, the loss of oxygen-containing groups is confirmed by XPS spectrum. As shown in Table S1, after hydrothermal reaction, the ratio of $\mathrm{C} / \mathrm{O}$ decreases significantly, which is similar to the literature reported by Shi et al [32].

3.2 Electrochemical performance of GPCP-X.

To demonstrate the potential of GPCP-X electrodes for supercapacitor applications, we further evaluated their electrochemical performance in a three-electrode system using1 $\mathrm{M} \mathrm{KOH}$ as electrolyte. The experimental results are shown in Figure 6. The average specific capacitance was calculated from the CVs according to equation (1);

$$
\mathrm{C}=\frac{1}{m v \Delta V} \int i(V) d v
$$


where $\mathrm{C}$ is the specific capacitance, $\mathrm{m}$ is the mass of active materials, $\mathrm{v}$ is the potential scan rate, $\Delta \mathrm{V}$ is the potential window in the $\mathrm{CV}, \int i(V) d v$ is the integrated area of the whole $\mathrm{CV}$ loop.

It can be seen in Figure 6a that the area of the CV curve of the GPCP-50 hybrids is larger than those of the CNT/PANI and G-Py at the same scan rate, indicating a higher specific capacitance of the nanohybrids. The exceptionally high specific capacitance and superior rate capability of the multiphase composite over those binary composites imply that the CNT/PANI hybrids combined with the G-Py aerogel has a strong synergistic effect between the components. It can be suggested that the introduction of CNT/PANI into the G-Py structure provides synergistic effects on both the microscale and nanoscale level, i.e., it facilitates efficient electron transport from CNT/PANI to the G-Py. The interwoven porous structure ensures effective access of the electrolyte ions to such an ultralight active material, substantially reducing the dead volume. The GPCP nanocomposite has the longest discharge time, meaning the highest specific capacitance. Figure $6 \mathrm{c}$ show the comparison of specific capacitance of the various components of GPCP-X. The specific capacitance of the GPCP-50 electrodes $\left(350 \mathrm{~F} \mathrm{~g}^{-1}\right)$ is 5 times that of G-Py aerogel electrode $\left(69.8 \mathrm{~F} \mathrm{~g}^{-1}\right)$ and 2.2 times that of CNT/PANI electrodes $\left(162 \mathrm{~F} \mathrm{~g}^{-1}\right)$, which clearly demonstrate superior performance. Figure $6 \mathrm{~d}$ and e show the cyclic voltammetry (CV) and galvanostatic charge/discharge of GPCP-X nanohybrids. It can be observed that the area of the $\mathrm{CV}$ becomes larger and the discharge time becomes longer with increasing ANI concentration, which signifies higher specific capacitance. From Figure 6f, it can be seen that the shape of CV curves was maintained at high scan rates (up to $100 \mathrm{mv} \mathrm{s}^{-1}$ ), which implies efficient charge transfer and electrolyte diffusion within the 3D GPCP-50 nanohybrids. To further understand the superior performance of the GPCP-50, electrochemical impedance spectroscopy (EIS) analysis was performed. As shown in Figure 6g, the Nyquist plots show a small arc in the 
high frequency region and a straight line in the low frequency region for all samples. It can also be observed that the GPCP-50 electrode displays the smallest semicircle at high frequencies suggesting it has that lowest charge transfer resistance, and a transition to linearity at low frequency that exhibits an ideal capacitive behavior. From these, it can be inferred that the assembly of CNT/PANI on G-Py consisting of GPCP nanohybrids can increase the accessible surface area of electrode as well as reduce the ion diffusion length during the charge-discharge process. From Figure 6h, it indicates that GPCP-50 results in an improved capacitance compared to other GPCP-X samples with lower PANI content. Moreover, the specific capacitance GPCP50 displayed a high specific capacitance of $406 \mathrm{~F} \mathrm{~g}^{-1}$ at $1 \mathrm{mv} \mathrm{s}^{-1}$.

The long-term cycle stability is a crucial parameter for supercapacitor electrode materials. The cycle stability of GPCP-50 nanohybrids was measured by long-term charge/discharge cycling at a current density of $2 \mathrm{~A} \mathrm{~g}^{-1}$, as shown in Figure S3. 84\% of specific capacitance can be retained even after 2,000 cycles. Usually, the degradation in capacitance is ascribed to swelling and shrinkage, which induced gradually deterioration of the conductivity and volumetric changes [46]. As a result, the good stability of GPCP comes from the synergistic effect between aligned PANI nanorods on CNT and G-Py aerogel. To sum up, the outstanding performance of GPCP can be attributed to two key reasons. First, the 1D conductive CNT/PANI not only prevent the aggregation and restacking of graphene sheets in some extent but also locally improve the conductivity of the aerogels by providing conductive pathways through defects of graphene and bridging the neighboring graphene sheets. Second, the incorporation of conductive polymers into the G-Py aerogel can effectively improve their energy density due to the pseudocapacitance originating from conducting polymers. As shown in Figure 3, the morphology of PANI exhibits an interesting change related to the increase of the concentration of ANI. Apparently, the 
acanthine structure of PANI protrudes more with the increase of aniline concentration. So the high capacitance of GPCP is mainly due to the high content of CNT/PANI, which can provide more pesudocapacitance contribution.

\section{Conclusions}

In summary, we have demonstrated that the architectures of 3D graphene-pyrrole/carbon nanotube/polyaniline (GPCP) nanohybrids can be successfully prepared via a simple method. When the G-Py aerogel was dipped into the CNT/PANI solution, the CNT/PANI were sandwiched between layers of G-Py and served as spacers to create gaps between neighboring graphene sheets, which can effectively reduce the layer-to-layer stacking of G-Py sheets. The calculated specific capacitances in supercapacitors using the 3D GPCP-50 electrodes are 5 times that of G-Py aerogel and 2.2 times that of CNT/PANI - its two main components. The experimental results presented here are a proof of concept that the ideal electrode templates for supporting high-performance active materials are necessary to achieve excellent electrochemical properties. The methods described demonstrates a simple way to design new functional materials that can be used as electrode materials for lightweight and flexible energy storage devices.

\section{Acknowledgment}

We are grateful for the National Natural Science Foundation of China (No. 51173170, 21101141, 50955010, 20974102), the financial support from the Innovation Talents Award of Henan Province (114200510019), State Key Laboratory of Chemical Engineering (No. SKL-

ChE-13A04), and the Key program of science and technology (121PZDGG213) from Zhengzhou Bureau of science and technology. The authors also acknowledge the Australian National 
Fabrication Facility (ANFF) for provision of services and equipment access, and are grateful for the support of ARC DP140100401.

\section{Appendix A. Supplementary data}

Supplementary data associated with this article can be found, in the online version. 


\section{References}

[1] Xu J J, Wang K, Zu S Z, Han B H and Wei Z X 2010 Hierarchical nanocomposites of polyaniline nanowire arrays on graphene oxide sheets with synergistic effect for energy storage ACS Nano 4 5019-26

[2] Sheng K X, Sun Y Q, Li C, Yuan W J and Shi G Q 2012 Ultrahigh-rate supercapacitors based on eletrochemically reduced graphene oxide for ac line-filtering Sci. Rep. 2247

[3] Zhang L and Shi G Q 2011 Preparation of highly conductive graphene hydrogels for fabricating supercapacitors with high rate capability J. Phys. Chem. C 115 17206-12

[4] Kim K S, Zhao Y, Jang H, Lee S Y, Kim J M, Kim K S, Ahn J H, Kim P, Choi J Y and Hong B H 2009 Large-scale pattern growth of graphene films for stretchable transparent electrodes Nature 457 706-10

[5] Chen Z P, Ren W C, Gao L B, Liu B L, Pei S F and Cheng H M 2011 Three-dimensional flexible and conductive interconnected graphene networks grown by chemical vapour deposition Nat. Mater. 10 424-8

[6] Huang C C, Bai H, Li C and Shi G Q 2011 A Graphene oxide/hemoglobin composite hydrogel for enzymatic catalysis in organic solvents Chem. Commun. 47 4962-4

[7] Dong X C, Xu H, Wang X W, Huang Y X, Chan-Park M B, Zhang H, Wang L H, Huang W and Chen P 2012 3D Graphene-cobalt oxide electrode for high-performance supercapacitor and enzymeless glucose detection ACS Nano 6 3206-13

[8] Srinivas G, Zhu Y W, Piner R, Skipper N, Ellerby M and Ruoff R 2010 Synthesis of graphene-like nanosheets and their hydrogen adsorption capacity Carbon 48 630-5

[9] Niu Z Q, Chen J, Hng H H, Ma J, Chen X D and Leavening A 2012 A leavening strategy to prepare reduced graphene oxide foams Adv. Mater. 24 4144-50 
[10] Chen J F, Lang Z L, Xu Q, Zhang J N, Fu J W, Chen Z M and Novel A 2013 A novel method to fabricate discrete porous carbon hemispheres and their electrochemical properties as supercapacitors Phys. Chem. Chem. Phys. 15 17786-92

[11] Fu J W, Chen Z M, Xu Q, Chen J F, Huang X B and Tang X Z 2011 The production of porous carbon nanofibers from cross-linked polyphosphazene nanofibers Carbon 49 $1037-9$

[12] Xu G H, Wang N, Wei J Y, Lv L L, Zhang J N, Chen Z M and Xu Q 2012 Preparation of graphene oxide/polyaniline nanocomposite with assistance of supercritical carbon dioxide for supercapacitor electrodes Ind. Eng. Chem. Res. 51 14390-8

[13] Xu G H, Xu D D, J N Zhang, Wang K X, Chen Z M, Chen J F and Xu Q 2013 Controlled fabrication of PANI/CNF hybrid films: Molecular interaction induced various micromorphologies and electrochemical properties J. Colloid Interface Sci. 411 204-12

[14] Lang J W, Kong L B, Wu W J, Luo Y C and Kang L 2008 Facile approach to prepare loose-packed NiO nano-flakes materials for supercapacitors Chem. Commun. 35 4213-5

[15] Fan H S, Wang H, Zhao N, Zhang X L and Xu J 2012 Hierarchical nanocomposite of polyaniline nanorods grown on the surface of carbon nanotubes for high-performance supercapacitor Electrode J. Mater. Chem. 22 2774-80

[16] Liu M K, Miao Y E, Zhang C, Tjiu W W, Yang Z B, Peng H S and Liu T X 2013 Hierarchical composites of polyaniline-graphene nanoribbons-carbon nanotubes as electrode materials in all-solid-state supercapacitors Nanoscale 5 7312-20

[17] Jin Y H, Fang M and Jia M Q 2014 In Situ One-pot synthesis of graphene-polyaniline nanofiber composite for high-performance electrochemical capacitors Appl. Surf. Sci. 308 $333-40$ 
[18] Gui D Y, Liu C L, Chen F Y and Liu J H 2014 Preparation of polyaniline/graphene oxide nanocomposite for the application of supercapacitor Appl. Surf. Sci. 307 172-7

[19] Schroder U, Niessen J and Scholz F 2003 A Generation of microbial fuel cells with current outputs boosted by more than one order of magnitude Angew. Chem. Int. Ed. 42 2880-3

[20] Shao L, Jeon J W and Lutkenhaus J L 2013 Porous polyaniline nanofiber/vanadium pentoxide layer-by-layer electrodes for energy storage J. Mater. Chem. A 1 7648-56

[21] Wang L, Ye Y J, Lu X P, Wen Z B, Li Z, Hou H Q and Song Y H 2013 Hierarchical nanocomposites of polyaniline nanowire arrays on reduced graphene oxide sheets for supercapacitors Sci. Rep. 33568

[22] Kobayashi $\mathrm{T}$, Yoneyama $\mathrm{H}$ and Tamura $\mathrm{H} 1984$ Oxidative degradation pathway of polyaniline film electrodes J. Electroanal. Chem. 177 293-7

[23] Meng Y N, Wang K, Zhang Y J and Wei Z X 2013 Hierarchical porous graphene/polyaniline composite film with superior rate performance for flexible supercapacitors Adv. Mater. 25 6985-90

[24] Shen J L, Yang C Y, Li X W and Wang G C 2013 High-performance asymmetric supercapacitor based on nanoarchitectured polyaniline/graphene/carbon nanotube and activated graphene electrodes ACS Appl. Mater. Interfaces 5 8467-76

[25] Li Z F, Zhang H Y, Liu Q, Sun L L, Stanciu L and Xie J 2013 Fabrication of high surface-area graphene/polyaniline nanocomposites and their application in supercapacitors ACS Appl. Mater. Interfaces 5 2685-91 
[26] Ge J, Cheng G H and Chen L W 2011 Transparent and flexible electrodes and supercapacitors using polyaniline/single-walled carbon nanotube composite thin films Nanoscale 3 3084-8

[27] Niu Z Q et al 2012 A “skeleton/skin” Strategy for preparing ultrathin free-standing single-walled carbon nanotube/polyaniline films for high performance supercapacitor Electrodes Energy Environ. Sci. 5 8726-33

[28] Li Y, Fang Y Z, Liu H, Wu X M and Lu Y 2012 Free-standing 3D polyaniline-CNT/Nifiber hybrid electrodes for high performance supercapacitors Nanoscale 4 2867-9

[29] Hummers W S and Offerman R E 1958 Preparation of graphitic oxide J. Am. Chem. Soc. 80 1339-1339

[30] Kovtyukhova N I, Ollivier P J, Martin B R, Mallouk T E, Chizhik S A, Buzaneva E V and Gorchinskiy A D 1999 Layer-by-layer assembly of ultrathin composite films from micron-sized graphite oxide sheets and polycations Chem. Mater. 11 771-8

[31] Zhao Y, Hu C G, Hu Y, Cheng H H, Shi G Q and Qu L T 2012 A versatile, ultralight, nitrogen-doped graphene framework Angew. Chem. 124 11533-7

[32] Xu Y X, Sheng K X, Li C and Shi G Q 2010 Self-assembled graphene hydrogel via a one-step hydrothermal process ACS Nano 4 4324-30

[33] Wu Z S, Sun Y, Tan Y Z, Yang S B, Feng X L and Mullen K 2012 Three-dimensional graphene-based macro- and mesoporous frameworks for high-performance electrochemical capacitive energy storage J. Am. Chem. Soc. 134 19532-5

[34] Lian P C, Zhu X F, Liang S Z, Li Z, Yang W S and Wang H H 2010 Large reversible Ccapacity of high quality graphene sheets as an anode material for lithium-ion batteries Electrochim. Acta 55 3909-14 
[35] Fan H S, Wang H, Zhao N, Zhang X L and Xu J 2012 Hierarchical nanocomposite of polyaniline nanorods grown on the surface of carbon nanotubes for high-performance supercapacitor Electrode J. Mater. Chem. 22 2774-80

[36] Wei J Y, Zhang J N, Liu Y, Xu G H, Chen Z M and Xu Q 2013 Controlled growth of whisker-like polyaniline on carbon nanofibers and their long cycle life for supercapacitors RSC Adv. 3 3957-62

[37] Marcano D C, Kosynkin D V, Berlin J M, Sinitskii A, Sun Z Z, Slesarev A, Alemany L B, Lu W and Tour J M 2010 Improved synthesis of graphene oxide ACS Nano 4 4806-14

[38] Xia X F, Hao Q L, Lei W, Wang W J, Sun D P and Wang X 2012 Nanostructured ternary composites of graphene/ $\mathrm{Fe}_{2} \mathrm{O}_{3} /$ polyaniline for high-performance supercapacitors $J$. Mater. Chem. 22 16844-50

[39] Pouget J P, Jdzefowiczt M E, Epstein A J, Tang X and MacDiarmid A G 1991 X-ray structure of polyaniline Macromolecule 24 779-89

[40] Lu X J, Dou H, Yang S D, Hao L, Zhang L J, Shen L F, Zhang F and Zhang X G 2011 Fabrication and electrochemical capacitance of hierarchical graphene/polyaniline/carbon nanotube ternary composite film Electrochim. Acta 56 9224-32

[41] Yoon J C, Lee J S, Kim S I, Kim K H and Jang J H 2013 Three-dimensional graphene nano-networks with high quality and mass production capability via precursor-assisted chemical vapor deposition Sci. Rep. 31788

[42] Yakuphanoglu F, Yahia I S, Barim G and Senkal B F 2010 Double-walled carbon nanotube/polymer nanocomposites: Electrical properties under dc and ac fields Synth. Met. 160 1718-26 
[43] Li J H, An J W, Zhou Y C, Ma Y X, Li M L, Yu M and Li S M 2012 Preparation of an amide group-connected graphene-polyaniline nanofiber hybrid and its application in supercapacitors ACS Appl. Mater. Interfaces 4 2870-6

[44] Yan X B, Chen J T, Yang J, Xue Q J and Miele P 2010 Fabrication of free-standing, electrochemically active and biocompatible graphene oxide-polyaniline and graphenepolyaniline hybrid papers ACS Appl. Mater. Interfaces 2 2521-9

[45] Sheng Z H, Shao L, Chen J J, Bao W J, Wang F B and Xie X H 2011 Catalyst-free synthesis of nitrogen doped graphene via thermal annealing graphite oxide with melamine and its excellent electrocatalysis ACS Nano 5 4350-8

[46] Wang Y G, Li H Q and Xia Y Y 2006 Ordered whiskerlike polyaniline grown on the surface of mesoporous carbon and its electrochemical capacitance performance $A d v$. Mater. 18 2619-23

[47] Li X et al 2013 Large-area flexible core-shell graphene/porous carbon woven fabric films for fiber supercapacitor electrodes Adv. Funct. Mater. 23 4862-9

[48] Cao Y C et al 2013 Boosting supercapacitor performance of carbon fibres using electrochemically reduced graphene oxide additives Phys. chem. chem. Phys. 15 19550-6

[49] Zang X B et al 2014 Highly flexible and adaptable, all-solid-state supercapacitors based on graphene woven-fabric film electrodes Small 10 2583-8

[50] Meng C Z, Liu C H and Fan S S 2009 Flexible carbon nanotube/polyaniline paper-like films and their enhanced electrochemical properties Electrochem. Commun. 11 186-9 


\section{Figure captions:}

Figure 1. Schematic illustration of the fabrication process: the assembly of $3 \mathrm{D}$ graphenepyrrole/carbon nanotube/polyaniline architectures.

Figure 2. Morphological characterizations and FTIR spectra of the nanocomposites. (a) Photographs of G-Py hydrogel (left) prepared with a $2 \mathrm{mg} \mathrm{mL}^{-1}$ homogeneous aqueous dispersion via hydrothermal reduction at $180^{\circ} \mathrm{C}$ for $12 \mathrm{~h}$ and G-Py aerogel obtained by freezedrying (right); (b) The SEM image and (c) TEM image of G-Py aerogel; (d) FTIR spectra of the Py, PPy and G-Py aerogel.

Figure 3. SEM images of CNT/PANI nanocomposites with different ANI concentration (a, d) 50mM; (b) 30mM; (c) 40mM.

Figure 4. SEM and TEM images of GPCP nanohybrids. The nanohybrids achieved with different concentration of ANI: (a, d) 50mM; (b) 30mM; (c) $40 \mathrm{mM}$.

Figure 5. X-ray diffraction spectra, Raman spectra and XPS of the nanohybrids. (a) XRD patterns of the CNT, G-Py, GO, neat PANI and GPCP-50 hybrid samples; (b) Raman spectra of pristine CNT, Graphene, G-Py, neat PANI, and GPCP-50 hybrids; XPS survey scan (c) and High-resolution N1s peaks (d) of the G-Py aerogel.

Figure 6. Electrochemical performance of GPCP nanohybrids. (a) Cyclic voltammetry at 5mv s ${ }^{-1}$ and (b) Galvanostatic charge/discharge curves of the G-Py, CNT/PANI and GPCP-50, at a current density of $1 \mathrm{~A} \mathrm{~g}^{-1}$; (c) Relational graph of specific capacitance to different nanomaterials, and the schematics representation of the microstructures of different nanomaterials; (d) Cyclic voltammetry at $5 \mathrm{mv} \mathrm{s}^{-1}$ and (e) Galvanostatic charge/discharge curves of the GPCP-X, at a current density of $1 \mathrm{~A} \mathrm{~g}^{-1}$; (f) Cyclic voltammetry curves of the GPCP-50; (g) Nyquist impedance plots of GPCP-30, 40, 50. (h) The variation of specific capacitances with different scan rates for GPCP-X. 


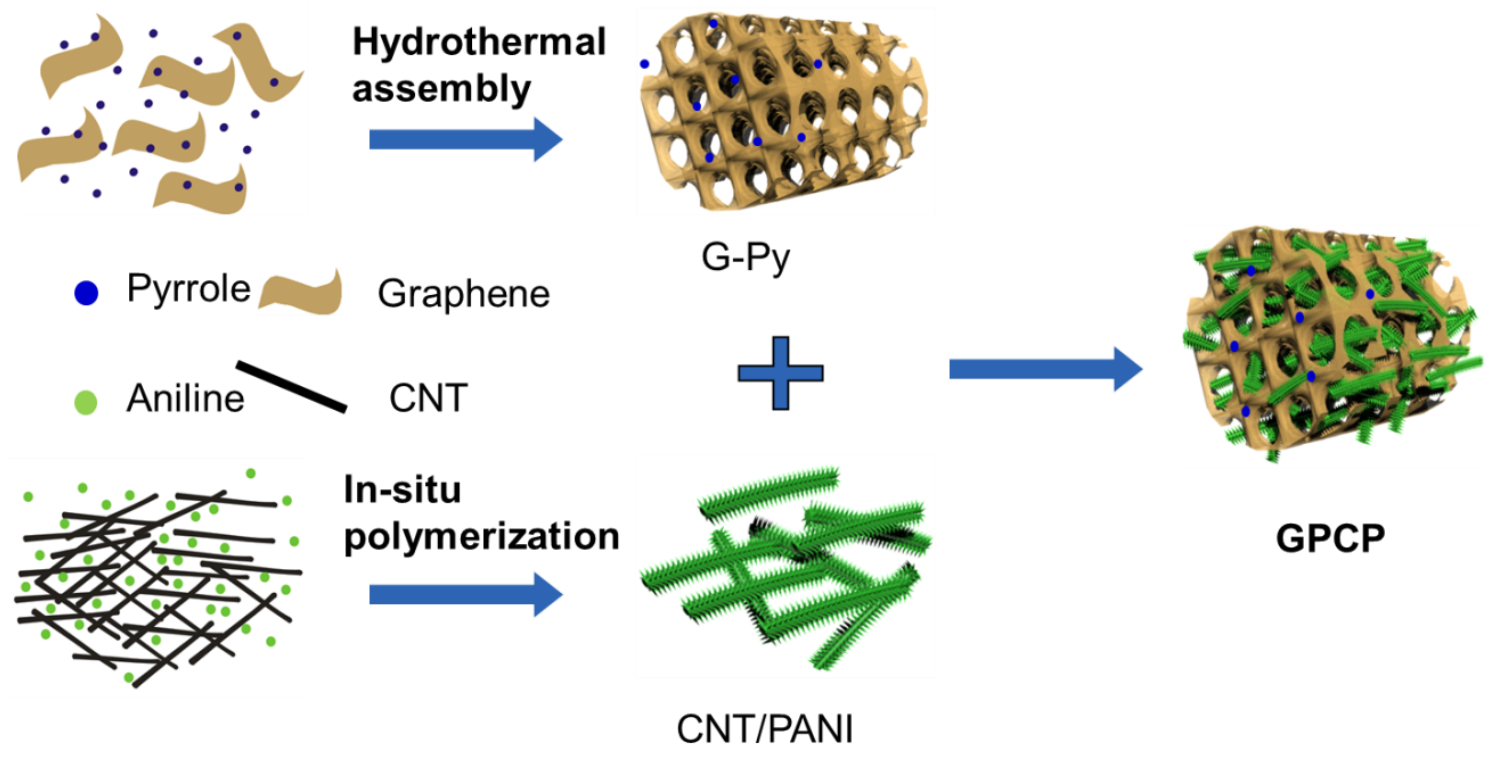

Figure 1. 

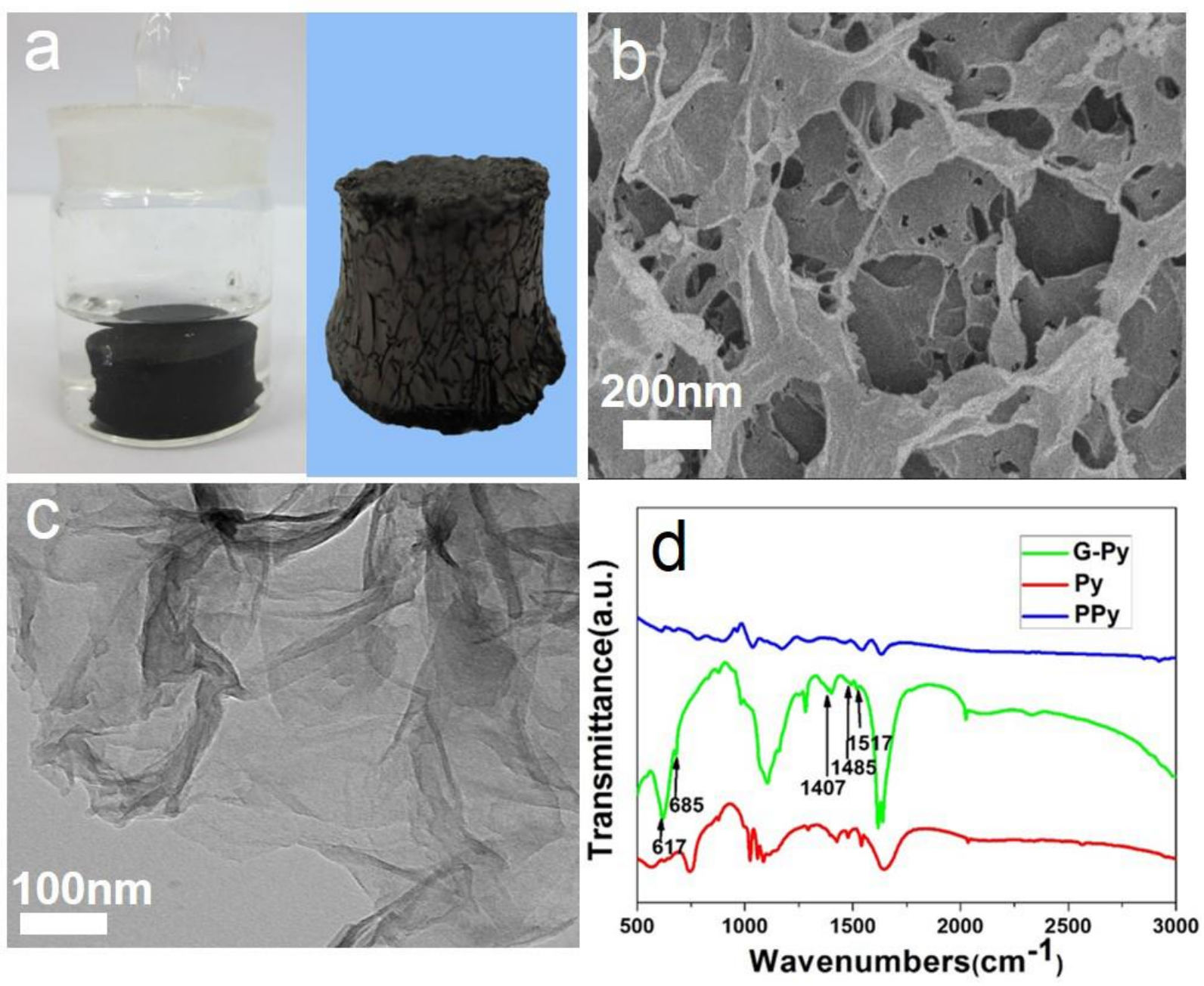

Figure 2. 

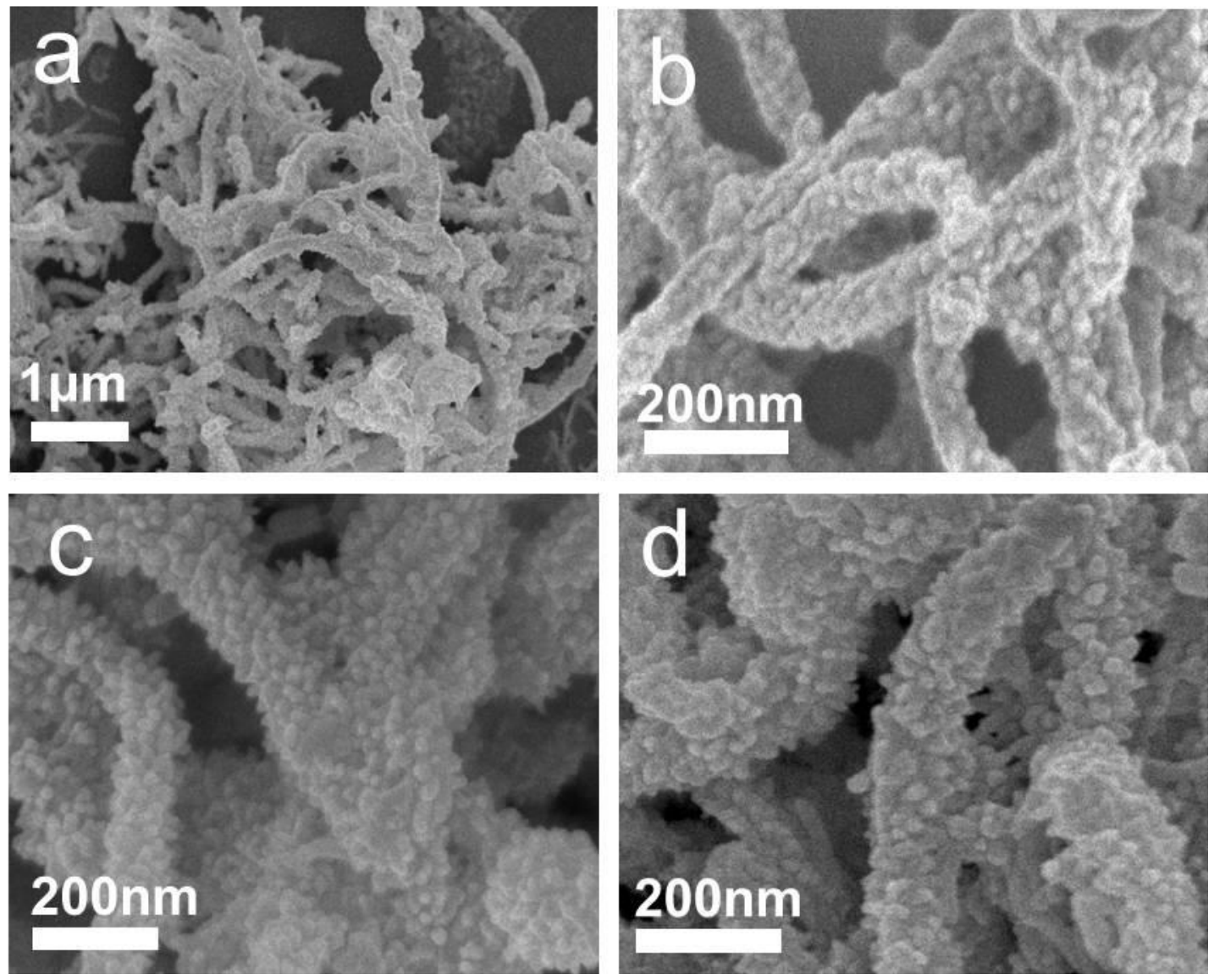

Figure 3. 

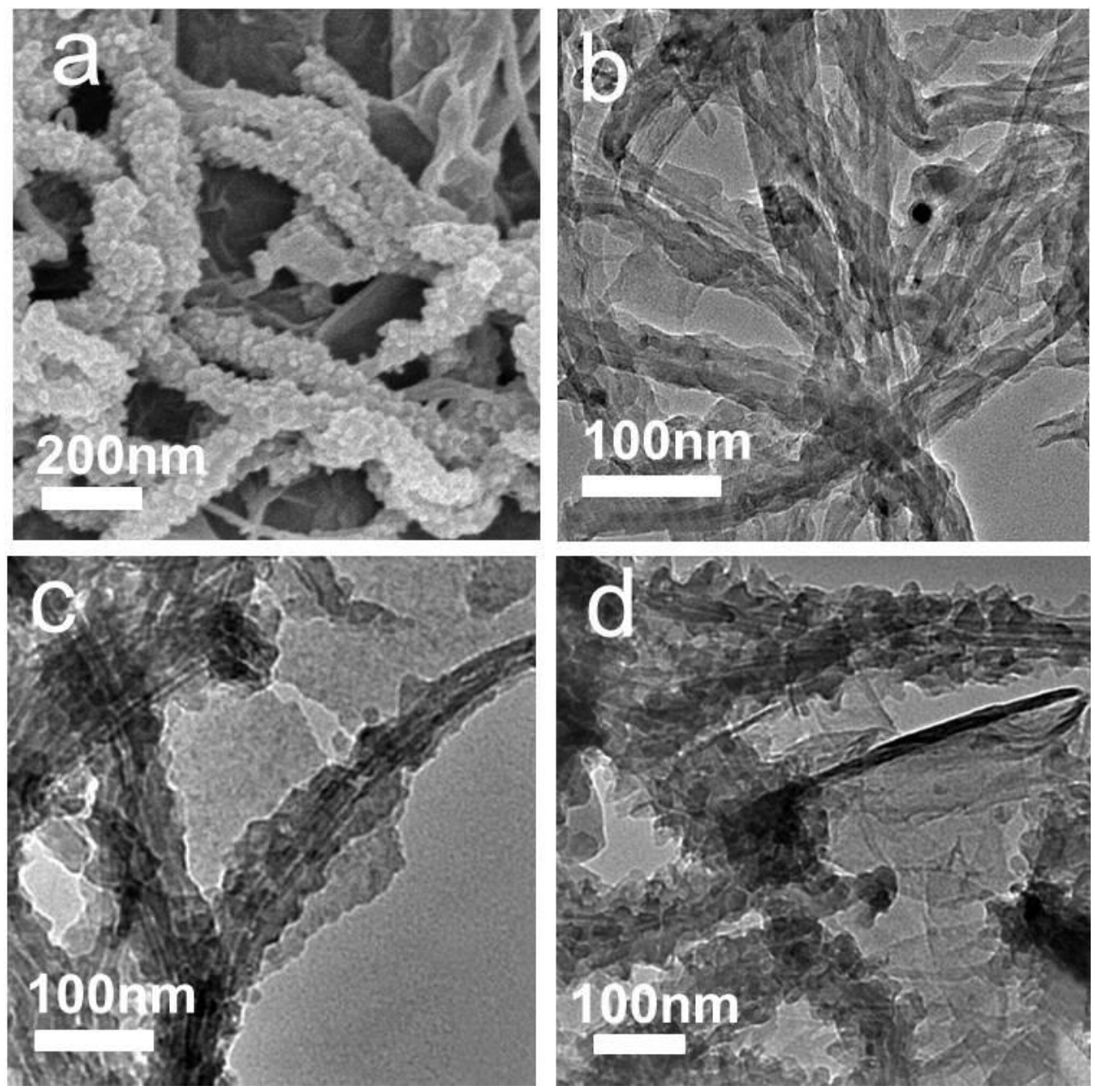

Figure 4. 

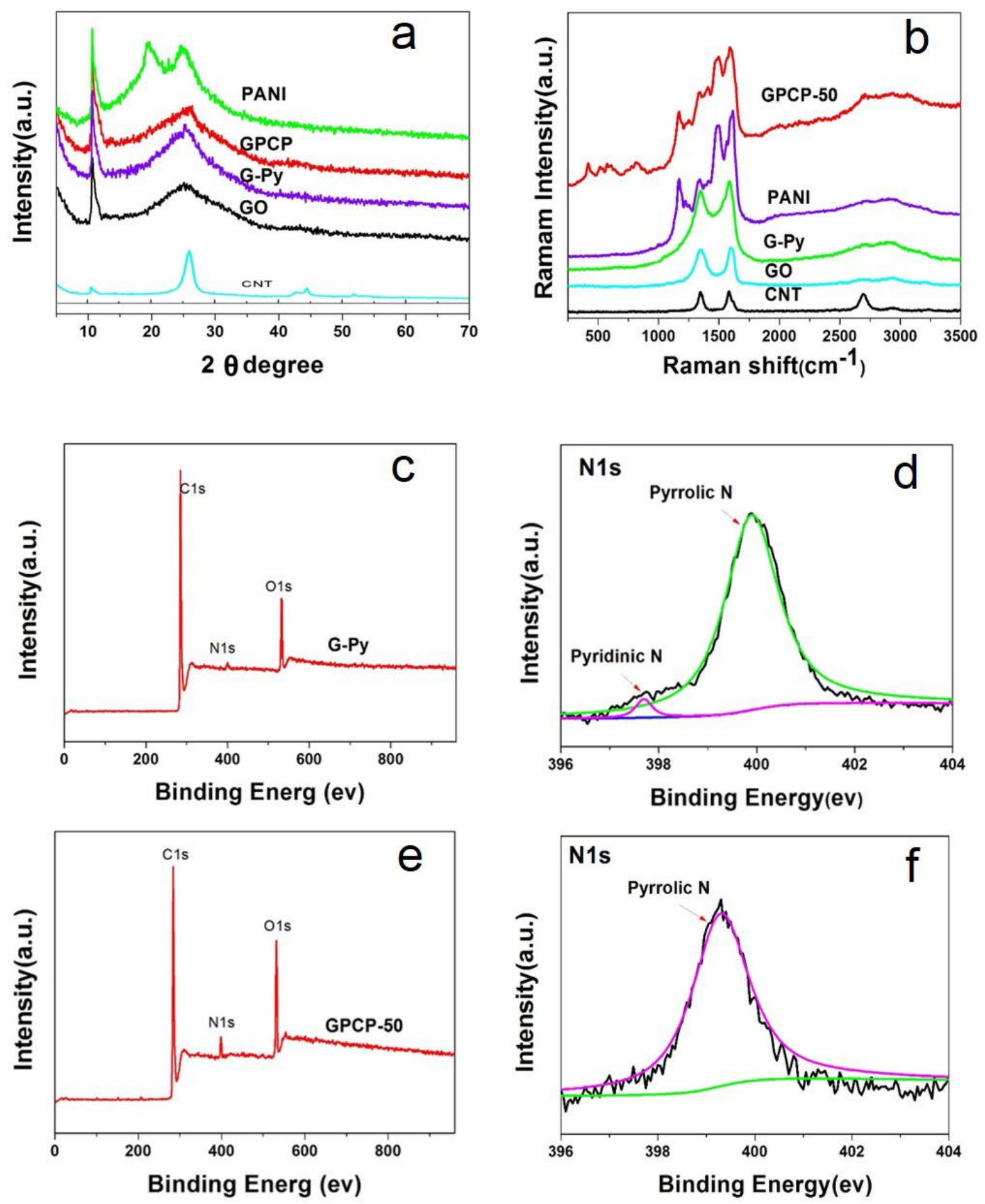

Figure 5. 

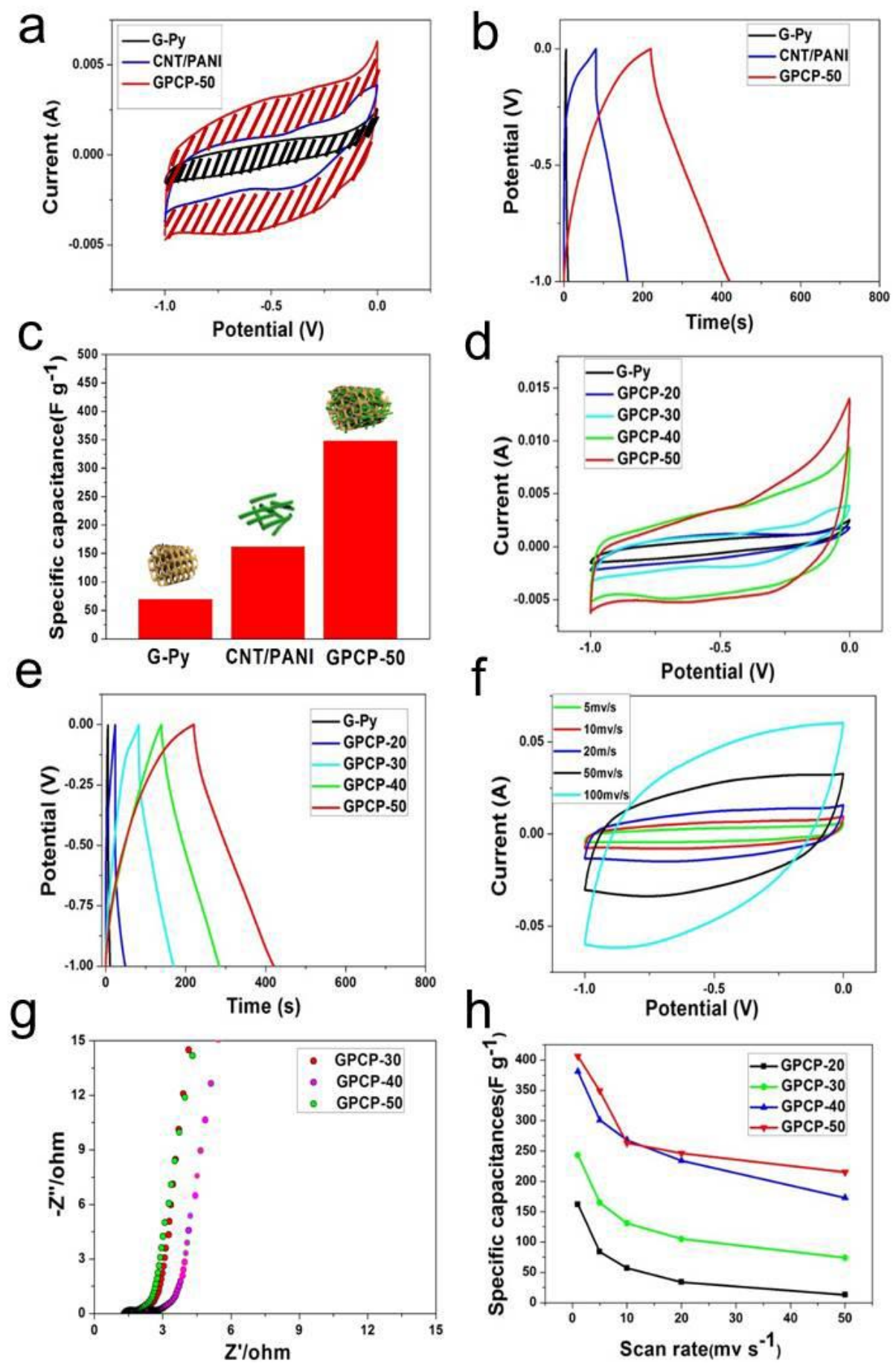

Figure 6. 
Graphical abstract
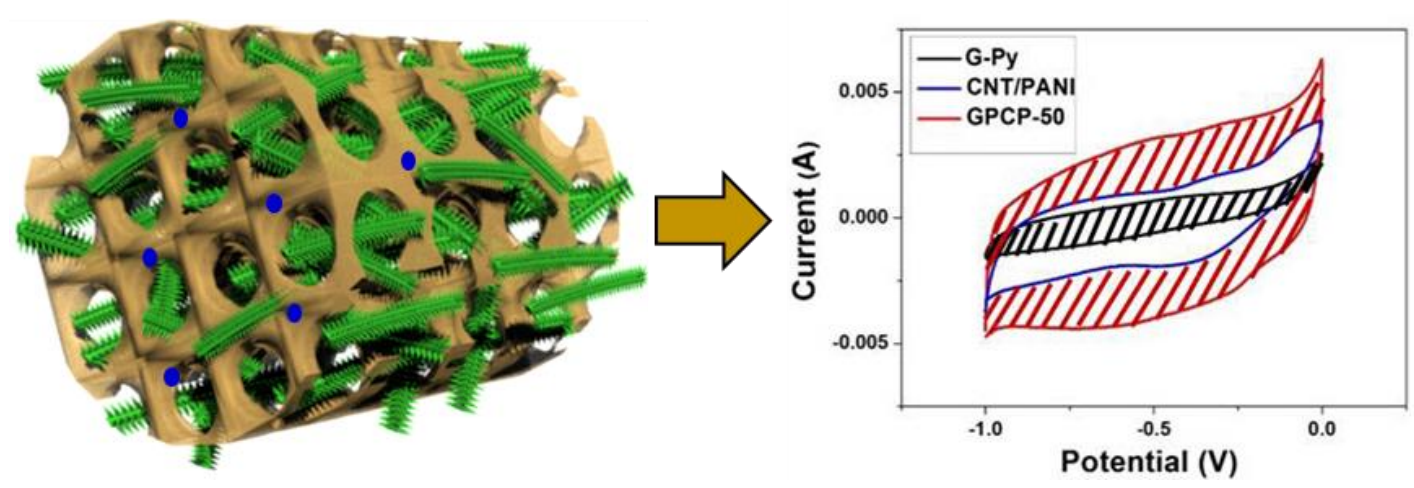

3D GPCP architectures for High-Performance Supercapacitor Applications 